\title{
Comparison of the Poly(vinyl alcohol) Adsorption Behaviour on the Mixed Oxides with Different Surface Structure
}

\author{
Małgorzata WIŚNIEWSKA ${ }^{1}{ }^{*}$, Katarzyna SZEWCZUK-KARPISZ ${ }^{1}$, Iwona OSTOLSKA ${ }^{1}$, \\ Konrad TERPILOWSKI ${ }^{2}$, Dariusz STERNIK ${ }^{3}$, Vladimir ZARKO ${ }^{4}$, Vladimir GUN'KO $^{4}$ \\ ${ }^{1}$ Department of Radiochemistry and Colloid Chemistry, Faculty of Chemistry, Maria Curie-Sklodowska University, \\ M. Curie-Sklodowska Sq. 3, 20-031 Lublin, Poland \\ ${ }^{2}$ Department of Interfacial Phenomena, Faculty of Chemistry, Maria Curie-Sklodowska University, M. Curie-Sklodowska \\ Sq. 3, 20-031 Lublin, Poland \\ ${ }^{3}$ Department of Physicochemistry of Solid Surface, Faculty of Chemistry, Maria Curie-Sklodowska University, M. Curie- \\ Sklodowska Sq. 3, 20-031 Lublin, Poland \\ ${ }^{4}$ Institute of Surface Chemistry, 17 General Naumov Street, 03164 Kiev, Ukraine \\ cross $^{\text {ref }}$ http://dx.doi.org/10.5755/j01.ms.22.2.7778
}

Received 18 August 2014; accepted 19 February 2015

\begin{abstract}
The adsorption and electrokinetic properties, as well as the stability of the mixed oxide suspensions in the absence and presence of poly(vinyl alcohol)-PVA were studied. Two alumina-silica-titania (AST) oxides were used: AST50 $\left(\mathrm{TiO}_{2}: 50 \%, \mathrm{SiO}_{2}: 28 \%, \mathrm{Al}_{2} \mathrm{O}_{3}: 22 \%\right)$ and $\mathrm{AST} 71\left(\mathrm{TiO}_{2}: 71 \%, \mathrm{SiO}_{2}: 8 \%, \mathrm{Al}_{2} \mathrm{O}_{3}: 21 \%\right)$. All measurements were carried out in the $p H$ range 3-9 as a function of the polymer molecular weight (72 000 and $100000 \mathrm{Da}$ ) and degree of PVA hydrolysis $(86 \%$ and $98 \%$ ). The obtained results indicated that the PVA adsorption strongly depends on the solution $p H$ - for AST50 it decreases and for AST71 increases with the $p H$ rise. This is caused by various PVA chains conformation on the solid surfaces with different structures. The adsorption of poly(vinyl alcohol) influences on the stability of the mixed oxides suspension. The greatest effects were observed in the presence of PVA 100000 at $p H 3$ for AST50 suspension and at $p H 9$ for AST71 system. In these cases, polymer addition leads to significant improvement of stability conditions of examined systems.

Keywords: alumina-silica-titania oxide, poly(vinyl alcohol), polymer adsorption, macromolecules conformation, suspension stability.
\end{abstract}

\section{INTRODUCTION}

The adsorption process of various substances (low molecular and macromolecular) $[1-4]$ is very desirable for many practical applications. The most important of them are: production of cosmetics, pharmaceuticals, paint, paper, food processing, drinking water purification, wastewater treatment, flotation, mineral processing, agriculture (reduction of cultivated soil erosion) and oil recovery [5-12]. The practical usage of polymers as stabilizers or flocculants of colloidal suspensions requires basing investigations. These experiments are designed to determine the structure and thickness of the polymer adsorption layer, which directly influences the stability conditions of such systems.

Stability of highly dispersed systems is conditioned not only by the amount of adsorbed polymer, but mainly on the conformation of macromolecules both adsorbed on the solid surface and nonadsorbed in the bulk solution (depletion interaction). The process of macromolecules adsorption on the solid surface is complex and depends on many factors including molecular weight and polydispersity of the polymer, $\mathrm{pH}$, ionic strength and temperature of the solution, as well as degree of purity of polymer samples and nature of the solid surface. Even a small change in one of these parameters can have a big impact on the suspension stability. Among above

\footnotetext{
* Corresponding author. Tel.: +48 815375622; fax: +48 815332811 .

E-mail address: wisniewska@hektor.umcs.lublin.pl (M. Wisniewska)
}

mentioned factors, influencing polymer chains conformation, one of the most important is the type of the solid surface groups. The precise characteristics of solid surface, including its charge, type and concentration of active sites, roughness, reactivity, as well as surface energy is essential for the determination of adsorbent-adsorbat interactions. They are responsible for the mechanism of polymer binding and stabilization-flocculation properties of the solid suspension.

For this reason, the aim of this study was to compare the effect of poly(vinyl alcohol) adsorption on the stability of mixed oxide suspensions differing with surface groups composition. They consist of three mineral oxides, such as alumina, silica and titania. Their percentage content determines the adsorption properties of the solid particles in relation to different substances (inorganic ions, natural and synthetic polymers, copolymers).

Additionally, these materials have significantly different properties and structure in comparison to the starting materials. There are a few methods of mixed oxides synthesis: sol-gel technique, high-temperature hydrolysis, chemical vapour deposition (CVD) and others [13]. Mixed oxides are widely used as components of ceramics, as catalysts for organic synthesis, adsorbents for various gases and toxic ions [14-17].

Applied poly(vinyl alcohol) is nonionic synthetic polymer characterized by good solubility in water, total biodegradability, excellent biocompatibility and nontoxicity. PVA has great usage in food industry as a binding and coating agent to retain the overall satisfactory taste, 
texture and quality of the food. Moreover, it is a very popular component of cements, varnishes, stabilizers of paints, pharmaceutical thickeners, as well as protective gloves, surgical threads, foils, plates and pipes resistant to benzene and oils [18-20].

\section{MATERIALS AND METHODS}

Two mixed alumina-silica-titania oxides (called AST) were used as adsorbents in the experiments. AST50 consists of: $50 \% \mathrm{TiO}_{2}, 28 \% \mathrm{SiO}_{2}$ and $22 \% \mathrm{Al}_{2} \mathrm{O}_{3}$, whereas AST71-71\% TiO $2,8 \% \mathrm{SiO}_{2}$ and $21 \% \mathrm{Al}_{2} \mathrm{O}_{3}$. These oxides were prepared in the Institute of Surface Chemistry of National Academy of Sciences of Ukraine in Kiev by chemical vapour deposition method - CVD. The average particle size of AST50 was $51 \mathrm{~nm}$ and AST71-24 nm. Their specific surface areas, determined by the BET method, are equal to 38 and $74 \mathrm{~m}^{2} / \mathrm{g}$, respectively [13].

Poly(vinyl alcohol) - PVA, of two molecular weights (72 000, $100000 \mathrm{Da})$ was used as the adsorbate. The PVA 72000 degree of hydrolysis is $97.5 \%$, whereas the PVA 100000 degree of hydrolysis is $86 \%$. This means that $2.5 \%$ (PVA 72000 ) or $14 \%$ (PVA 100000 ) of acetate groups in the PVA macromolecules do not undergo hydrolysis to hydroxyl ones. The acetate groups in the PVA chains adopt negative charge due to the fact, that $\mathrm{C}-\mathrm{H}$ bonds located at $\alpha$ position relative to the acetate groups are of acidic properties. The proton from the $-\mathrm{CH}_{2}$ - group can dissociate and in this way a resonant structure is formed:

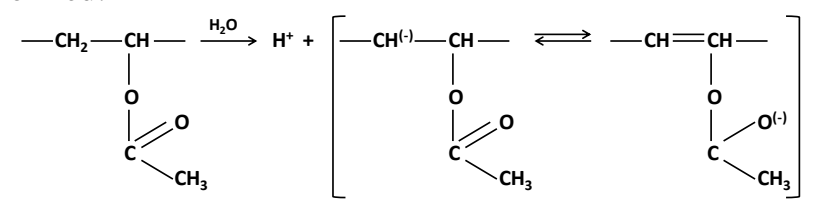

All measurements were performed at $25^{\circ} \mathrm{C}$, as a function of solution $\mathrm{pH}(3,6$ or $9 \pm 0.1)$. $\mathrm{NaCl}$ with the concentration of $0.01 \mathrm{~mol} / \mathrm{dm}^{3}$ was used as a supporting electrolyte.

The PVA adsorption amount was determined from the difference in the polymer concentration before and after the adsorption process. The poly(vinyl alcohol) concentration was determined using its reaction with $\mathrm{H}_{3} \mathrm{BO}_{3}$ and $\mathrm{I}_{2}$ solutions [21]. The intensity of the obtained green colour was measured spectrophotometrically with UV-Vis spectrophotometer Carry 100 (Agilent Technologies). The adsorption measurements were carried out at the PVA concentration $100 \mathrm{ppm}$ using $0.025 \mathrm{~g}$ AST50 and $0.015 \mathrm{~g}$ of AST71.

Potentiometric titration method was applied to determine the solid surface charge density [22]. The Titr_v3 computer program developed by W. Janusz was used for this purpose. The measuring set was consisted of: thermostat RE 204 (Lauda), glass and calomel electrode (Backman Instruments), pHmeter PHM 240 (Radiometer), automatic microburette Dosimat 765 (Metrohm) and computer. Initially, the supporting electrolyte solution and the AST suspensions (containing $0.29 \mathrm{~g}$ of AST50 and $0.1 \mathrm{~g}$ of AST71) without PVA were titrated with $\mathrm{NaOH}$ $\left(0.1 \mathrm{~mol} / \mathrm{dm}^{3}\right)$. Then, the AST suspensions in the presence of PVA (100 ppm) were titrated in analogous way. Zeta potential measurements were performed using a zetameter Zetasizer Nano-ZS (Malvern Instruments) equipped with a titrator allowing automatic solution $\mathrm{pH}$ determination during the measurement. Zeta potential of solid particles was measured in the absence and presence of PVA $(100 \mathrm{ppm})$. The samples were prepared by the addition of $0.003 \mathrm{~g}$ of the mixed oxide to $50 \mathrm{~cm}^{3}$ of the appropriate solution (supporting electrolyte or polymer).

Stability measurements of the AST suspensions in the absence and presence of PVA were made using a turbidimeter Turbiscan Lab ${ }^{\text {Expert }}$ with a cooling module TLab Cooling. The suspensions without polymer were prepared by the addition $0.002 \mathrm{~g}$ of the solid to the $\mathrm{NaCl}$ solution or PVA solution (100 ppm). Each system was subjected to sonification for 3 minutes. The polymer was added just before the measurement started. The single measurement lasted 15 hours during which the relevant data was recorded every 15 minutes. The results were obtained as the curves of transmission and backscattering of light passing through the sample during the measurement. The TSI factor (Turbiscan Stability Index) was calculated by the computer software from the following formula:

$T S I=\sqrt{\frac{\sum_{i=1}^{n}\left(x_{i}-x_{B S}\right)^{2}}{n-1}}$,

where $x_{i}$ is the average backscatterring for each minute of measurement; $x_{B S}$ is the average $x_{i}$ value, $n$ is the scans number.

\section{RESULTS AND DISCUSSION}

As can be seen in Fig. 1 and Fig. 2, the amount of the adsorbed PVA on the AST surface clearly depends on the solution $p H$. For AST50 the decrease of poly(vinyl alcohol) adsorption with the increasing $p H$ is observed. The opposite behavior was obtained for AST71 systems: the lowest adsorption level was observed at $p H 3$, whereas the highest - at $p H$ 9. These trends are visible for both PVA 72000 and PVA 100 000. This is probably caused by the specific conformation of the adsorbed polymer chains on the surfaces of examined oxides. The structure of PVA adsorption layer is determined by the interaction of polymer segments with the different types of active sites on the solid surface.

The adsorbed amounts of PVA depends on the degree of polymer acetate groups ionization, as well as the sign and magnitude of the solid surface charge. On the one hand, the PVA chains contain a certain amount of acetate groups, which may be a source of negative charge. Despite their small content in the macromolecules $(2.5 \%-\mathrm{PVA}$ 72000 and $14 \%$-PVA 100000 ), they have a strong impact on the polymer chain conformation. The degree of ionization of the acetate groups increases with the $p H$ rise leading to development of the PVA chains. The lower the degree of hydrolysis is, the higher the number of negatively charged acetate groups in PVA macromolecules is. As a consequence, the PVA 100000 chains adopt more extended conformation than the PVA 72000 chains. On the other hand, the point of zero charge $\left(p H_{p z c}\right)$ of the AST50 particles is 4.8 and for AST71 it is equal to 4.2 (Table 1). 
Table 1. Electrokinetic parameters for AST systems with and without PVA, $C_{P V A}=100 \mathrm{ppm}$

\begin{tabular}{|c|c|c|c|c|c|c|}
\hline \multirow{2}{*}{ Parameter } & \multicolumn{3}{|c|}{ AST50 } & \multicolumn{3}{c|}{ AST71 } \\
\cline { 2 - 7 } & - & PVA 72000 & PVA 100000 & - & PVA 72000 & PVA 100000 \\
\hline$p H_{p z c}$ & 4.8 & 4.8 & 4.8 & 4.2 & 4.2 & 4.2 \\
\hline$p H_{\text {iep }}$ & 5.8 & 3.6 & 3.4 & 8.6 & 9.0 & 9.0 \\
\hline
\end{tabular}

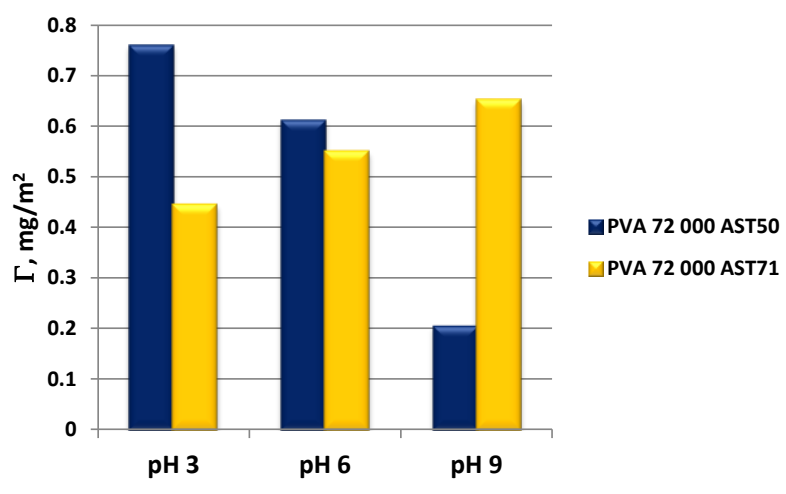

Fig. 1. Adsorbed amounts of PVA 72000 on the AST50 and AST71 surfaces at different solution $p H, C_{P V A}=100 \mathrm{ppm}$

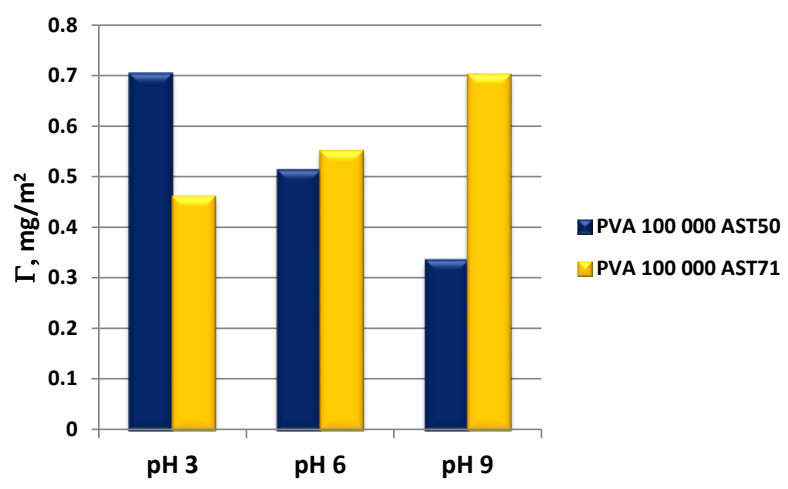

Fig. 2. Adsorbed amounts of PVA 100000 on the AST50 and AST71 surfaces at different solution $p H, C_{P V A}=100 \mathrm{ppm}$

It means that at $p H<p H_{p z c}$ the mixed oxide surface has positive charge, whereas at $p H>p H_{p z c}$ the adsorbent surface is negatively charged.The adsorption of PVA does not change the $p H_{p z c}$ position and also the solid surface charge density (Table 1).The obtained results confirm that the most probable interactions of the polymer with the surface are hydrogen bridges. No major change in the concentration of potential-creating ions in the surface layer indicates, that primarily polymer hydroxyl groups are involved in the hydrogen bonds formation. These groups do not undergo ionization and therefore are not a source of electric charges.

Nevertheless, the specific conformation of the adsorbed macromolecules is responsible for changes of PVA adsorption on the AST surfaces with the increasing $p H$ (for AST50 - adsorption decrease, for AST71 adsorption increase). It is influenced mainly by the type and concentration of the hydroxyl surface groups of the mixed oxide, both neutral (-MeOH) and charged $\left(-\mathrm{MeOH}_{2}{ }^{+},-\mathrm{MeO}^{-}\right)$, where $\mathrm{Me}$ means $\mathrm{Al}, \mathrm{Si}$ and Ti atoms.

At $p H 3$ the polymer chains adopt the least expanded conformation due to negligible ionization of acetate groups. The highest adsorption of PVA on the AST50 surface at $p H 3$ indicates that polymer macromolecules are bounded with the solid active sites in the form of polymer coils. These coils must be densely packed in the surface layer ensuring the high adsorption level. In turn, the PVA macromolecules probably form on the AST71 surface the loop and tail structures of small length (thin adsorption layer). In such situation, the adsorbed single chain occupies a substantial part of the mixed oxide surface, blocking access of other adsorbate macromolecules and leading to the lowest level of PVA adsorption at $p H 3$. It should be also noted, that at $p H 3$ the both examined adsorbents has a small positive surface charge. Thus, the electrostatic attraction between the adsorbent and the PVA macromolecules (having a small negative charge) occurs.

At $p H \quad 6$ the intermediate adsorption level was observed for both examined mixed oxides. Under these conditions the polymer macromolecules adopt a more extended conformation than at $p H 3$. This is caused by the increase in the degree of acetate group ionization and rising repulsion of the charged PVA segments. Additionally, the adsorbate-adsorbent repulsion occurs. As a result, the structure of the adsorbed PVA chains is characterized by a larger length of the loops and tails formed on the solid surface.

At $p H 9$ the polymer macromolecules contain only charged acetate groups. As a consequence, their development is the greatest under such $p H$ conditions. In the case of AST50 systems the solid surface charge density reaches values from -10 to $-15 \mu \mathrm{C} / \mathrm{cm}^{2}$, whereas for AST71 this value is $-30 \mu \mathrm{C} / \mathrm{cm}^{2}$. Under these conditions macromolecules adopt the most extended conformation on the AST71 surface, so that the maximal PVA amount can adsorb on the unit of solid surface. The electrostatic repulsion between PVA chains and AST50 surface groups is significantly weaker and the polymeric adsorption layer must be flatter (than on the AST71 surface). This makes the adsorption of great number of polymer macromolecules impossible due to blockade of the solid active sites. As a result, the lowest poly(vinyl alcohol) adsorption on the AST 50 surface at $p H 9$ was observed.

The presence of the adsorbent - adsorbate repulsion in the examined systems at $p H 6$ and 9 indicates the specific nature of the PVA adsorption. This means that the hydrogen bridges are main interactions responsible for poly(vinyl alcohol) adsorption under these $\mathrm{pH}$ conditions. The hydroxyl groups of the PVA macromolecules and the surface groups of the adsorbent (both ionized and neutral) are involved in their formation.

The adsorption of poly(vinyl alcohol) influences the elektrokinetic properties of AST oxides (Fig. 3 and Fig. 4). In the case of AST 50, in the $p H$ range 3-6 the zeta potential reduction in the polymer presence (in comparison to system without PVA) is observed. At $p H>6$ the increase of electrokinetic potential of the system containing polymer takes place.

For AST71 systems, the adsorption of poly(vinyl alcohol) reduces the electrokinetic potential of the solid 
particles in the range of $p H 3-8$ and increases in the range of 9-10. A difference between the values of $p H_{p z c}$ and $p H_{\text {iep }}$ (Table 1), especially large in the case of AST71 particles, is related to the porosity of the adsorbent. Diffusion parts of the electric double layers, formed on the pore surface, probably overlap each other, resulting in specified $\mathrm{pH}_{\text {iep }}$ value.

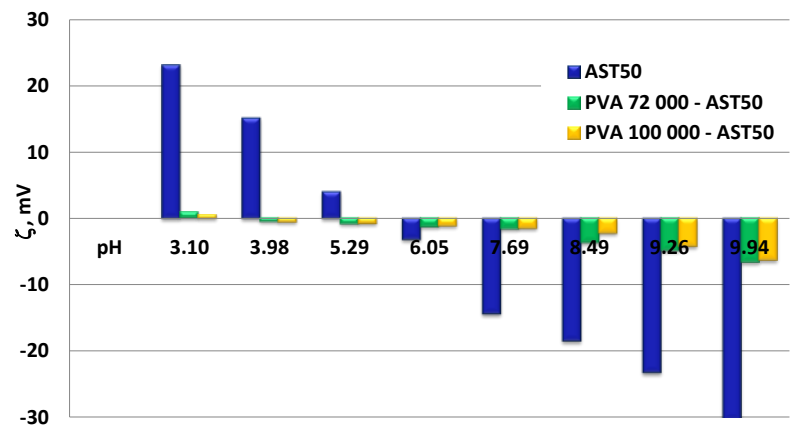

Fig. 3. Zeta potential of the AST50 particles with and without PVA at different solution $p H, C_{P V A}=100 \mathrm{ppm}$

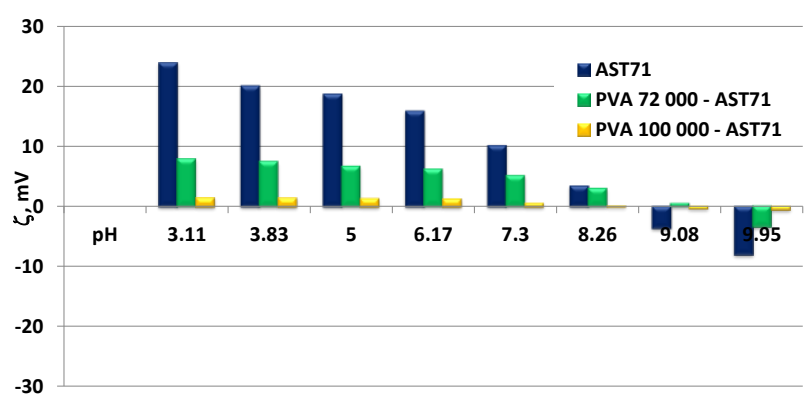

Fig. 4. Zeta potential of the AST71 particles with and without PVA at different solution $p H, C_{P V A}=100 \mathrm{ppm}$

The observed decrease in the zeta potential is mainly caused by the shift of slipping plane causing by the adsorbed polymer chains. The PVA macromolecules take a specific conformation with some loops and tails on the adsorbent surface. These structures are responsible for substantial shift of the slipping plane from the solid surface and the $\zeta$ potential reduction. Additionally, the decrease in the electrokinetic potential is also associated with negatively charged acetate groups present in the PVA chains. If these groups are located in the diffusion part of the electrical double layer of the solid particles, they reduce zeta potential of the system. The observed changes in the electrokinetic potential values can also be connected with the effect of the surface active site blocking by the adsorbed polymer macromolecules. As a result, these sites become inaccessible to electrolyte ions and other PVA chains. This causes changes in the charge distribution in the diffusion layer, thereby influencing the electrokinetic potential value. The larger number of segments connected directly with the adsorbent surface is, the greater number of the supporting electrolyte counterions is pushed from the surface layer to the diffusion one. Observed value of electrokinetic potential is the summary result of all these effects contribution.

The poly(vinyl alcohol) addition causes significant changes of the mixed oxide suspension stability (Fig. 5). Analyzing of the TSI coefficients, one should note, that the higher its value is, the more unstable examined system is.
The TSI changes in the range from 0 (highly stable systems) to 100 (dramatically unstable suspensions).

The AST50 suspension without PVA is relatively stable at $p H 3$, whereas at $p H 6$ and 9 its stability clearly deteriorates.

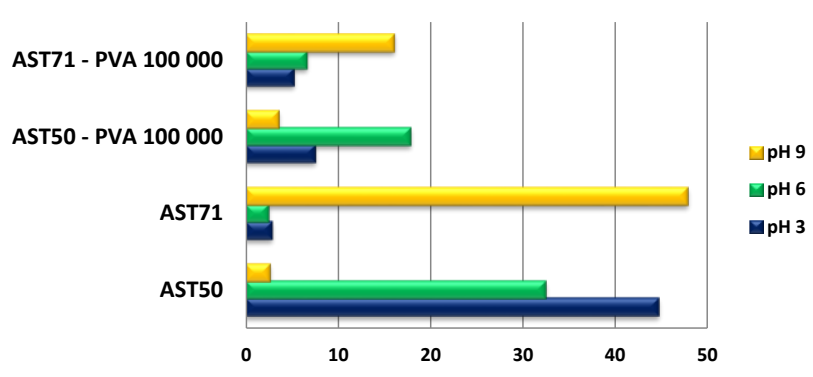

Fig. 5. TSI coefficients for AST systems with and without PVA 100000 at different solution $p H, C_{P V A}=100 \mathrm{ppm}$

On the other hand, the AST71 system without polymer is stable at $p H$ of 3 and 6 , and at $p H 9$ it is unstable. Too low zeta potential values (or close to zero) are responsible for destabilization process of investigated systems. Thus, the small strength or even lack of repulsive electrostatic forces between the particles leads to their effective collisions and the formation of aggregates.

The addition of PVA 100000 to AST50 suspension improves significantly its stability at $p H 6$ and 9. At $p H 3$ the stability conditions in the system with polymer is similar to the system without it (suspension remains relatively stable). The highly packed PVA layer consisted of polymeric coils adsorbed on the AST50 surface at $p H 3$ ensures effective steric stabilization of the suspension. The increasing degree of poly(vinyl alcohol) ionization with the rise of $\mathrm{pH}$ and more and more extended conformation of adsorbed macromolecules favours the electrosteric mechanism of AST50 system stability in the polymer presence (especially at $p H$ 9).

Adsorption of poly(vinyl alcohol) with molecular weight 100000 does not cause significant changes in the stability of the AST71 suspension at $p H 3$ and $6-$ the examined systems are rather stable due to the steric or electrosteric repulsion. In turn, at $p H 9$ the dramatic increase in the suspension stability as a result of the PVA addition is observed. The presence of extremely developed polymer chains in the adsorption layer, reduces significantly the contact possibility of the particles coated with the polymer. Moreover, the total ionization of PVA macromolecules enhances this effect due to electrostatic repulsion of solid particles covered with polymeric layer (electrosteric stabilization).

\section{CONCLUSIONS}

The effect of the poly(vinyl alcohol) adsorption on the electrokinetic properties and suspension stability of two mixed oxides of alumina-silica-titania was examined. The obtained results showed that the percentage content of individual compounds $\left(\mathrm{Al}_{2} \mathrm{O}_{3}, \mathrm{SiO}_{2}\right.$ and $\left.\mathrm{TiO}_{2}\right)$ has the big impact on the adsorption properties of applied solids in relation to the PVA. Polymer adsorption amount on the mixed oxides surfaces varies with the solution $\mathrm{pH}$ value. For AST50 the decrease of poly(vinyl alcohol) adsorption 
with the increasing $p H$ is observed, whereas the opposite behavior was obtained for AST71 systems. The specific conformation of the adsorbed macromolecules is responsible for this. It is influenced by both the type and concentration of the hydroxyl surface groups of the mixed oxide, and ionization degree of acetate groups in PVA macromolecules. The greatest impact on the AST50 and AST71 suspensions stability has the polymer at $p H 3$ and 9, respectively. At these $p H$ values significant improvement of system stability is observed (steric and electrosteric mechanisms of stabilization).

\section{Acknowledgments}

The research leading to these results has received funding from the People Programme (Marie Curie Actions) of the European Union's Seventh Framework Programme FP7/2007-2013/ under REA grant agreement n'PIRSESGA-2013-612484.

\section{REFERENCES}

1. Nosal-Wiercińska, A. Adsorption of Cystine at Mercury/ Aqueous Solution of Chlorate (VII) Interface in Solutions of Different Water Activity Central European Journal of Chemistry 10 2012: pp. 1290-1300.

2. Nosal-Wiercińska, A., Grochowski, M. Adsorption of Thiourea and Its Methyl Derivatives from Chlorate (VII) with Varied Water Activity Collection of Czechoslovak Chemical Communications 76 2011: pp. 265-275.

3. Ostolska, I., Wiśniewska, M. Comparison of the Influence of Polyaspartic Acid and Polylysine Functional Groups on the Mechanism of Polymeric Film Formation at the $\mathrm{Cr}_{2} \mathrm{O}_{3}-$ Aqueous Solution Interface Applied Surface Science 311 2014: pp. $734-739$.

http://dx.doi.org/10.1016/j.apsusc.2014.05.149

4. Grządka, E., Mendrek, B., Chibowski, S., Wiśniewska, M., Trzebicka B. Investigations of the Properties of the Manganese Dioxide Suspension in the Presence of Guar Gum and Carboxymethyl Ccellulose Materials Chemistry and Physics 144 2014: 361-368.

5. Tripathy, T., De, B. R. Flocculation: a New Way to Treat the Waste Water Journal of Physical Science 19 2006: pp. $93-127$.

6. Gurumoorthy, A. V. P., Kha, K. H. Polymers at Interfaces: Biological and Non-biological Applications Recent Research in Science and Technology 3 2011: pp. 80-86.

7. Roberts, J. C. Paper Chemistry. Chapman\&Hall, London, 1996.

8. Sojka, R. E., Bjorneberg, D. L., Entry, J. A., Lentz, R. D., Orts, W. J. Polyacrylamide in Agriculture and Environmental Land Management Advanced Agronomy 92 2007: pp. $75-162$.

9. Tadros, T. F. Colloids Aspects of Cosmetic Formulations with Particular Reference to Polymeric Surfactants Colloids and Interface Science Series 4 2008: pp. 1-34.
10. Shahidi, F., Arachchi, J. K. V., $\quad$ Yeon Y. J. $\quad$ Food Application of Chitin and Chitosans Trends in Food Science and Technology 10 1999: pp. 37-51. http://dx.doi.org/10.1016/S0924-2244(99)00017-5

11. Ji, Y. Z., Zhang, Y. F., Chen, X. P., Gao, H. S. Application of Organic Polymeric Flocculants in Centrifugal Dewatering of Oil Refinery Sludge Journal of Environmental Science 15 2003: pp. 510-513.

12. Moody, G. The Use of Polyacrylamides in Mineral Processing Mineral Engineering 5 1992: pp. 479-492.

13. Gun'ko, V. M., Zarko, V. I., Mironyuk, I. F., Goncharuk, E. V., Guzenko, N. V., Borysenko, M. V., Gorbik, P. P., Mishchuk, O. A., Janusz, W., Leboda, R., Skubiszewska-Zięba, J., Grzegorczyk, W., Matysek, M., Chibowski, S. Surface Electric and Titration Behavior of Fumed Oxides Colloids and Surfaces A 240 2004: pp. 9-25.

14. Gawande, M. B., Pandey, R. K., Jayaram, R. V. Role of Mixed Metal Oxides in Catalysis Science-Versatile Applications in Organic Synthesis Catalysis Science and Technology 2 2012: pp. 1113-1125.

15. Guidelli, E. J., Guerra E. M., Mulato, M. Vanadium and Titanium Mixed Oxide Films: Synthesis, Characterization and Application as Ion Sensor Journal of Electrochemical Society 159 2012: pp. J217-J222.

16. Kaciulis, S., Pandolfi, L, Viticoli, S, Sberveglieri, G, Zampiceni, E, Wlodarski, W, Galatsis, K, L Y.X. Investigation of Thin Films of Mixed Oxides for Gas-Sensing Applications Surface and Interface Analysis 34 2002: pp. $672-676$.

17. Weilong, W, Xiaobo, F. Efficient Removal of $\mathrm{Cr}(\mathrm{VI})$ with Fe/Mn Mixed Metal Oxide Nanocomposites Synthesized by a Grinding Method Journal of Nanomaterials ID 514917 2013: pp. $1-8$.

18. Baker, M. I., Walsh, S. P, Schwartz, Z., Boyan, B. D. A Review of Polyvinyl Alcohol and Its Uses in Cartilage and Orthopedic Applications Journal of Biomedical Materials Research B Applied Biomaterials $\quad 100$ 2012: pp. $1451-1457$.

19. Goodship, V., Jacobs, D. Polyvinyl Alcohol: Materials, Processing and Applications (Rapra Review Reports), Smithers Rapra Press, 2009.

20. Jiang, T., Wang, G., Qiu, J., Luo, L., Zhang, G. Preparation and Biocompatibility of Polyvinyl Alcohol Small Intestinal Submucosa Hydrogel Membranes Journal of Medical and Biological Engineering 29 2009: pp. 102107.

21. Zwick, M. M. Poly(vinyl alcohol)-Iodine Complexe Journal of Applied Polymer Science 9 1965: pp. $2393-2424$. http://dx.doi.org/10.1002/app.1965.070090706

22. Janusz, W. Electrical Double Layer at the Metal Oxide/Electrolyte Interface in Interfacial Forces and Fields: Theory and Applications Surfactant Science 85 M. Dekker, New York, 1999. 